\title{
Axial Domain Wall Dimension in Bistable Glass-Coated Microwire
}

\author{
M. Kladivová*, J. Ziman and P. Duranka \\ Department of Physics, Technical University of Košice, Park Komenského 2, 04200 Košice, Slovakia
}

\begin{abstract}
The properties of the so-called head-to-head domain wall in a bistable glass-coated microwire are studied. The stray field produced by this wall affects the shape of the voltage peaks induced in the pick-up coils in the SixtusTonks experiment. If the shape of these peaks is to be used to obtain information about the wall geometry, the pickup coil parameters (length and radius) have to be taken into account. In the experiment presented in this paper, the two pairs of pick-up coils are used. The coils differ only in their radii, which means that differences between the induced peaks in the coils are only caused by the stray field. Comparison of theoretical and experimental voltage peaks provides information about shortening of the axial dimension of the propagating domain wall with increasing applied axial magnetic field. This effect is more visible when a pair of pick-up coils with smaller radii is used in the experiment. Preliminary analysis and comparison of the signals indicate that the proposed method could help in understanding the mechanism of unidirectional effect.
\end{abstract}

DOI: 10.12693/APhysPolA.137.855

PACS/topics: bistable glass-coated microwires, magnetic domain wall, Sixtus-Tonks experiment

\section{Introduction}

Bistable glass-coated microwires provide a unique opportunity to study the dynamics of a single domain wall between axial domains. In order to understand the detailed mechanism of these processes, knowledge about the domain wall structure or geometry is necessary [1-3]. One of the possible way to obtain information about the wall properties is to analyse the signal induced in the pick-up coil in the standard Sixtus-Tonks experiment $[4,5]$. However, the situation is complicated by the fact that the so-called head-to-head domain wall in bistable glass-coated microwires is not standard $180^{\circ}$ domain walls, because the normal to the wall component of magnetization is not equal to zero. This kind of domain wall is the source of a stray field which significantly influences the parameters of induced voltage peaks $[2,6]$. In this paper we show how the shape of induced voltage peaks combined with measurement of domain wall velocity can provide information about changes in the wall geometry. A brief analysis of induced peaks obtained using two pairs of identical pick-up coils with different radii is also presented.

\section{Experimental}

The standard Sixtus-Tonks experiment was used to measure the domain wall velocity. Two pairs of pick-up coils were used with the same length $d=0.5 \mathrm{~mm}$, and number of turns $n=29$, but differing in their radii, i.e., $\quad R_{1}=0.15 \mathrm{~mm}$ and $R_{2}=1.05 \mathrm{~mm}=7 R_{1}$ (Fig. 1 ). Voltage peaks induced in the pick-up coils were analysed.

*corresponding author; e-mail: maria.kladivova@tuke.sk (a)
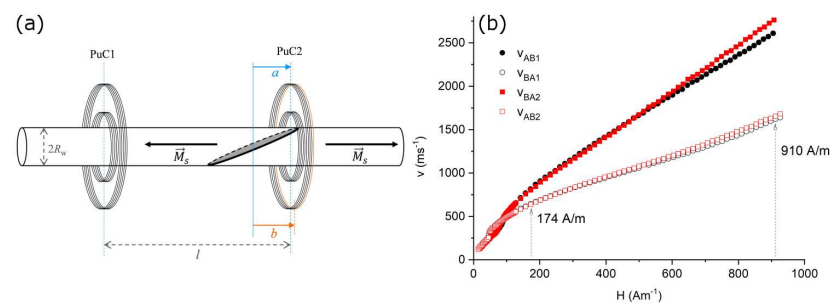

Fig. 1. (a) Schematic diagram of the system with two pairs of pick-up coils at distance $l$ and of the sample with a single planar domain wall of length $L$. (b) Four wall velocities versus magnitude of applied field dependencies.

A detailed description of the experimental set-up enabling measurement of four different velocities and detection of the presence of the so-called unidirectional effect can be found in $[7,8]$.

Measurements were carried out on an $\mathrm{Fe}_{77.5} \mathrm{Si}_{15} \mathrm{~B}_{7.5}$ glass-coated microwire prepared by means of the TaylorUlitovski method. The following parameters were chosen: radius of metallic core $R_{w}=7.5 \mu \mathrm{m}$, thickness of glass coating $7.5 \mu \mathrm{m}$, and length of the sample $12.5 \mathrm{~cm}$. For the experiment presented in this paper we chose a piece of microwire exhibiting strong unidirectional behaviour $[7,8]$. This fact is reflected by the dependencies in Fig.1. The four velocities in this figure satisfy the condition $v_{\mathrm{AB} 1}(H) \approx v_{\mathrm{BA} 2}(H) \neq v_{\mathrm{AB} 2}(H) \approx v_{\mathrm{BA} 1}(H)$.

\section{Results and discussion}

As discussed recently [2], the shape of the signal is influenced by the shape of the domain wall, as well as by the presence of a stray field which accompanies the moving wall. In a bistable microwire, the voltage induced in each pick-up coil $u_{\mathrm{pc}}$ can be expressed as 


$$
\begin{gathered}
u_{\mathrm{pc}}=-\frac{\mathrm{d} \Phi}{\mathrm{d} t}=-\frac{\mathrm{d}\left(\Phi_{w}+\Phi_{d}\right)}{\mathrm{d} t}= \\
-\frac{\mathrm{d}\left(\Phi_{w}+\Phi_{d}\right)}{\mathrm{d} a} \frac{\mathrm{d} a}{\mathrm{~d} t}=-\frac{\mathrm{d}\left(\Phi_{w}+\Phi_{d}\right)}{\mathrm{d} a} v,
\end{gathered}
$$

where $\Phi$ is the total magnetic flux through the winding of the pick-up coil, $v$ is the axial wall velocity, $\Phi_{w}$ is the axial magnetic flux due to magnetization inside domains, and $\Phi_{d}$ is the magnetic flux due to the stray field. The distance between centres of the wall and the pick-up coil is denoted as $a$. For the simple model of a planar domain wall (depicted in Fig. 1) the total magnetic flux $\Phi$ through the pick-up coil can be expressed as [2]

$$
\Phi=\Phi_{w}+\Phi_{d}=\frac{n}{d} \int_{a-d / 2}^{a+d / 2}\left(\Phi_{w 1}+\Phi_{d 1}\right) \mathrm{d} b,
$$

where $n$ is the number of turns in the pick-up coil, $d$ is the pick-up coil length, $b$ is the distance of one turn of the coil from the wall centre, and $\left(\Phi_{w 1}+\Phi_{d 1}\right)$ is the total axial magnetic flux through this coil turn. Since variable $a$ appears only in the integral boundaries, thus using (1) and (2) one can obtain

$$
\begin{aligned}
& \frac{u_{\mathrm{pc}}(a)}{v}=-\frac{d\left(\Phi_{w}+\Phi_{d}\right)}{\mathrm{d} a}= \\
& -\frac{n}{d}\left[\Phi_{w 1}\left(a+\frac{d}{2}\right)+\Phi_{d 1}\left(a+\frac{d}{2}\right)\right. \\
& \left.-\Phi_{w 1}\left(a-\frac{d}{2}\right)-\Phi_{d 1}\left(a-\frac{d}{2}\right)\right] .
\end{aligned}
$$

Note that if the moving wall does not change its shape (length), the quantity on the left side of (3) as a function of the wall position $a$ should not depend on the wall velocity. This clearly indicates that dependence $u_{\mathrm{pc}}(a) / v$ provides information about changes in the wall geometry.

We have modelled the right hand side of (3) for various planar wall lengths for both radii of the pick-up coils used in the experiment. The obtained model dependencies are depicted in Fig. 2. One can see then that the signal measured with small pick-up coils is more sensitive to changes in the wall length. Figure 2c shows the difference between signals from the two pickup coils. Since only $\Phi_{d 1}$ depends on the pick-up coil radius, the subtraction of signals from identical coils with different radii gives only a signal from a stray field. In Figs. 2c and 2d, this part of the signal is strongly influenced by the wall length $L$. These model dependencies could be used to estimate the wall length, as well as the changes in it as the maximum signal values (i.e., extreme in dependence $u_{\mathrm{pc}}(a) / v$ ) can be easily obtained from the experiment.

Typical experimental records of the first induced signal $\left(u_{p c} / v\right)$ peak were obtained using both pairs of pickup coils for $v_{\mathrm{AB} 2}$ dependence (see Fig. 1) at two values of external axial field $H_{1}=174 \mathrm{~A} / \mathrm{m}, H_{2}=910 \mathrm{~A} / \mathrm{m}$ $\left(H_{2} \approx 5 H_{1}\right)$, and are depicted as a function of a wall position in Fig. 3a. In agreement with the model

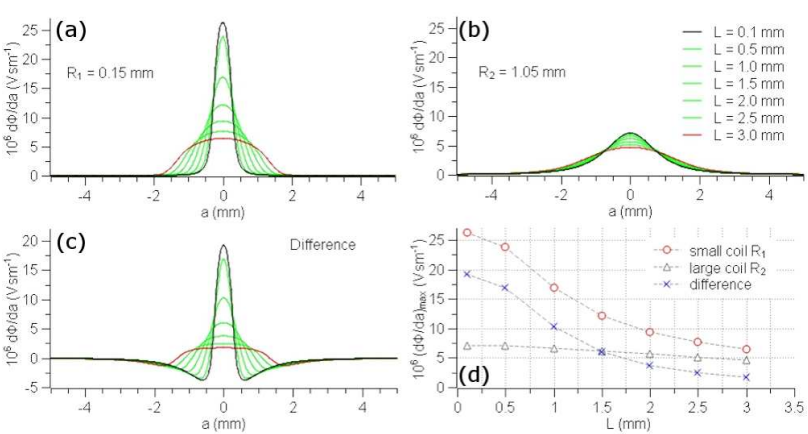

Fig. 2. (a-c) Model curves for function $\mathrm{d} \Phi / \mathrm{d} a$ for both pick-up coil radii, as well as the signal difference between them. (d) maximum values of dependencies $(\mathrm{a}-\mathrm{c})$ as a function of the wall length $L$.

dependencies presented in Fig. 2, the experimental signals obtained using small pick-up coils (red symbols) are higher and narrower than those obtained using the larger coils (black symbols). It is worth notice that the dependencies in Fig. 3a are functions only of the wall position with respect to the pick-up coil. Thus, as long as the wall does not change its length, there should not be observed difference in signals that were measured using the same coil for various values of magnetic field. However, significant differences can be observed in the experimental dependencies for different fields in Fig. 3a. For both pairs of coils, comparison of these dependences with the model ones in Fig. 2 shows that a faster wall (for $H_{2}$, empty symbols) is shorter than a slower one (for $H_{1}$, full symbols). This shortening of the wall qualitatively agrees with the theoretical prediction [2], that a wall moving at a higher velocity should be shorter due to its deformation (eddy currents produced by moving wall create an inhomogeneous damping field, which influences the wall shape). Comparison of the maximum values of theoretical and experimental peaks in Figs. 2, 3 suggests a shortening of the wall from $2 \mathrm{~mm}$ to $1.4 \mathrm{~mm}$. These values are too large, and do not satisfactorily explain the observed wall mobility (see model in [2]). This disagreement may be caused by the fact that the model dependencies were calculated for a planar wall, which could be applicable for a domain wall in low fields [9]. In fields higher than about $100 \mathrm{~A} / \mathrm{m}$ (above the sudden jump in $v(H)$ dependencies in Fig. 1), it is possible that the wall shape is different (for instance $[1,3,8]$ ). This assumption is also supported by the fact that the observed signals are not symmetrical.

This experiment can also provide some information about the mechanism of unidirectional effect. The shapes of the first peaks for both "slow" (red symbols, $v_{\mathrm{AB} 2}$ ) and "fast" (black symbols, $v_{\mathrm{AB} 1}$ ) branches of $v(H)$ dependencies in Fig. 1b can be compared, see in Fig. $3 \mathrm{~b}$ for a low value of external field $H_{1}$ (full symbols), and for a high value of $H_{2}$ (empty symbols). These measurements were performed using a pair of pick-up coils with small radius $R_{1}$. For low, as well as for high value of the applied 


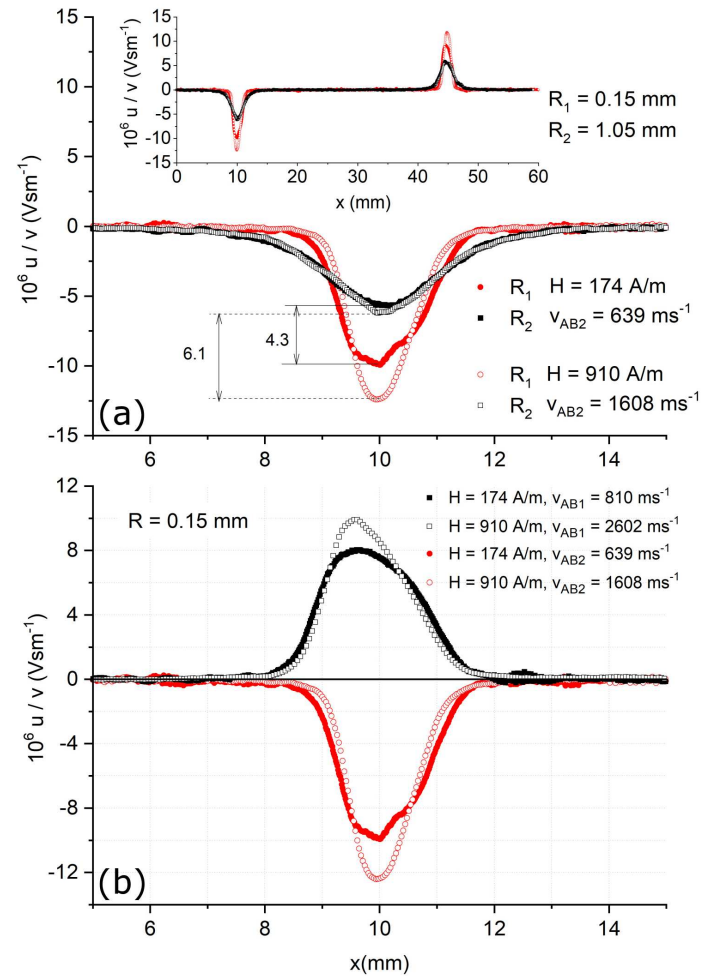

Fig. 3. (a) Details of the first induced signal $\left(u_{\mathrm{pc}} / v\right)$ peak obtained using both pairs of pick-up coils for $H_{1}$ and $H_{2}$ on a "slow" branch of $v(H)$ dependencies $\left(v_{\mathrm{AB} 2}\right)$ in Fig. 1 as a function of wall position $x$. The insert shows the complete signal record obtained in our Sixtus-Tonks experiments. (b) The first peak of induced signal $\left(u_{\mathrm{pc}} / v\right)$ obtained using the smaller-radius pick-up coil from the "slow" ( $\left.v_{\mathrm{AB} 2}\right)$, as well as the "fast" $\left(v_{\mathrm{AB} 1}\right)$ branch of $v(H)$ dependences in Fig. 1 for fields $H_{1}$ and $H_{2}$.

field the signal shape for the "slow" branch of dependencies in Fig. 1b is narrower and higher, i.e., the wall is shorter here, than the wall which is subject to motion on a "fast" branch for the same value of external field.

The observed behaviour supports the interpretation of unidirectional effect based on the mechanism proposed in [7]. This can be briefly explained as follows. For the same value of external field the damping force $F_{d}$ is the same for both "slow" and "fast" domain walls. Using the model of a planar wall $[8,10]$ we can write

$$
\begin{aligned}
& F_{d} \sim \frac{\beta+\tilde{\beta}_{e c, f}}{L_{f}} v_{f}=\frac{\beta+\tilde{\beta}_{e c, s}}{L_{s}} v_{s}, \\
& \tilde{\beta}_{e c, f, s} \cong \operatorname{const} \frac{1}{\rho_{f, s}},
\end{aligned}
$$

where $\beta$ is the damping coefficient due to the spin relaxation, $\tilde{\beta}_{e c}$ is the coefficient due to eddy currents damping, $\rho$ is resistivity, and indexes $f$ and $s$ denote "fast" and "slow" walls, respectively. Let us consider solid walls $\left(L_{f}=L_{s}\right)$, and let $\beta_{e c, f}<\tilde{\beta}_{e c, s}$. In this case $v_{f}>v_{s}$, and also $v_{f} \tilde{\beta}_{e c, f}<v_{s} \tilde{\beta}_{e c, s}$. The last relation indicates that eddy current damping is stronger for the "slow" wall, and it could be the reason why this wall, if it can change length, is shorter.

\section{Conclusions}

A new experiment with a new method of processing experimental data obtained in Sixtus-Tonks experiments is proposed. The presented method of experimental data processing can be used as a tool for detecting changes in the wall geometry from the signals induced in the pickup coils. Comparison of model and experimental dependencies confirmed the wall shortening with increasing velocity (increasing external magnetic field). This fact could explain the decrease in wall mobility with increasing field observed in glass-coated bistable microwires. Using two pairs of pick-up coils with different radii, we also found that the stray field significantly changes the shape of the induced signal, and that the signals from small pick-up coils are more suitable for data processing due to their greater sensitivity. The experiment was performed on a sample with strong unidirectional effect. Our preliminary analysis and comparison of the signals suggests that the proposed method may help in understanding the mechanism of unidirectional effect.

\section{Acknowledgments}

This research was supported by VEGA Grant No. 1/0388/18 from the Scientific Grant Agency of the Ministry for Education of the Slovak Republic and by the Slovak Research and Development Agency under contract No. APVV-16-0079.

\section{References}

[1] L. Panina, M. Ipatov, V. Zhukova, A. Zhukov, Physica $B$ 407, 1442 (2012).

[2] M. Kladivová, J. Ziman, J. Magn. Magn. Mater. 480, 193 (2019).

[3] R. Varga, P. Klein, A. Jimenez, M. Vazquez, Phys. Status Solidi A 213, 356 (2016).

[4] F. Beck, J.N. Rigue, M. Carara, J. Magn. Magn. Mater. 435, 21 (2017).

[5] S.A. Gudoshnikov, Yu.B. Grebenshchikov, B.Ya. Ljubimov, P.S. Palvanov, N.A. Usov, M. Ipatov, A. Zhukov, J. Gonzalez, Phys. Status Solidi A 206, 613 (2009).

[6] P.A. Ekstrom, A. Zhukov, J. Phys. D 43, 205001 (2010).

[7] J. Onufer, J. Ziman, M. Kladivová, J. Magn. Magn. Mater. 396, 313 (2015).

[8] J. Onufer, J. Ziman, P. Duranka, M. Kladivová, Physica B 540, 58 (2018).

[9] M. Kladivová, J. Ziman, J. Kecer, P. Duranka, Acta Phys. Pol. A 131, 639 (2017).

[10] D.X. Chen, N.M. Dempsey, M. Vazquez, A. Hernando, IEEE Trans. Magn. 31, 781 (1995). 